\title{
High Power Laser Processing with Ultrafast and Multi-Parallel Beams
}

\author{
A. Gillner ${ }^{1,2}$, J. Finger ${ }^{1}$, P. Gretzki ${ }^{1}$, M. Niessen ${ }^{1}$, Thilo Bartels ${ }^{1}$, M. Reininghaus ${ }^{1}$ \\ ${ }^{1}$ Fraunhofer-Institute for Laser Technology, Steinbachstrasse 15, 52074 Aachen, Germany \\ E-mail: arnold.gillner@ilt.fraunhofer.de \\ ${ }^{2}$ LLT Chair of Laser Technology, RWTH Aachen, Steinbachstrasse 15, 52074 Aachen, Germany
}

\begin{abstract}
High power ultrashort pulsed lasers are an ultimate manufacturing tool for a large variety of materials and provide outstanding properties for high precision manufacturing with almost no thermal effects and numerous new processing possibilities. However, using high power ultrashort pulsed lasers with high pulse repetition frequencies in the $\mathrm{MHz}$ region can cause thermal issues like overheating, melt generation and low ablation quality. High ablation quality only can be achieved, if all process parameters are carefully matched, which requires a deep understanding of the process, intensive simulation for selecting processing strategies and innovative system components. Beside ultra high speed scanning using polygon scanners the use of multiple laser beams provide the best and most versatile high power ablation solution. With switchable single beams using parallel acoustooptic and phase modulating beam steering systems together with diffractive optical beam splitter, high ablation rates can be achieved while maintaining the high processing quality. However, using multiple laser beams each single beam can influence the adjacent beam either by heat accumulation or by plasma and vapour emission. Distance of the single beams, pulse repetition frequency and scanning strategy have to be matched to the material and ablation geometry. With a careful adaption of all parameters highly accurate and fast processing can be achieved. With this approach a next step up to an all optical manufacturing system can be provided.
\end{abstract}

DOI: $10.2961 /$ jlmn.2019.02.0003

Keywords: ultrafast laser processing, multi beam processing, diffractive optical elements, beam splitting, high speed scanning, laser ablation, laser drilling, simulation

\section{Motivation}

The average power of commercial ultra-short pulsed laser sources is significantly increasing and thus this new tool becomes more and more interesting even for large scale applications [1-4]. Today, robust picosecond and femtosecond lasers with average powers of 50-150 W are used for cutting and ablation of sapphire and glass at high speed without losing quality of the ablation. However, when looking at typical ultrafast laser processes for metals and ceramics with high quality laser ablation, laser drilling or laser cutting, the average power used in most processes is in the range of 1 to several $10 \mathrm{~W}$ for a single beam processing with a classic galvanometric scanning system. With this approach 3D microstructuring of metal surfaces for tooling and printing applications with ps-lasers is boosted by the availability of new high power ultrashort pulse laser sources with high industrial performance allowing short processing times. Using this technology for large parts and high speed ablation the efficient utilization of the high average laser power in the field of material processing requires an effective distribution of the laser power onto the workpiece due to several effects in the ablation process. Therefore up to now despite the fact that machining with ultrashort laser pulses offer higher precision, the technology is still not being used in the industry for large scale parts as ultra-short pulsed laser (USP) ablation is still lacking productivity with an magnitude smaller ablation rate compared to the ns-laser pulse ablation.

The typical process parameters for some representative processes are shown in Fig. 1 and are arranged by pulse energy and repetition frequency. The majority of processes use low average power below 10W. Only a few processes (glass cutting, sapphire drilling and interference pattering) uses higher power and pulse energies. In most cases the repetition frequency is limited by heat accumulation and pulse-plasma interaction. So a naive scaling of USP processes by increasing the average power is limited or not efficient.

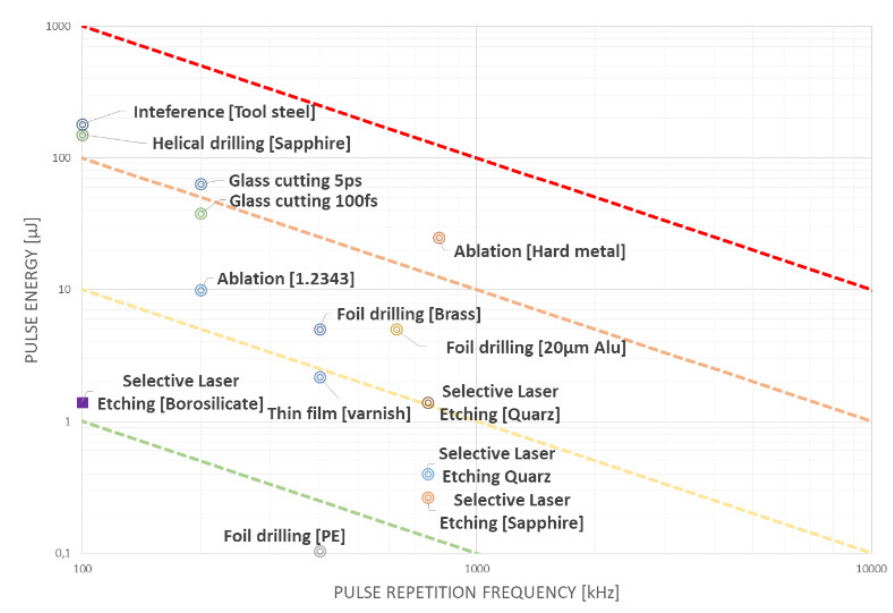

Fig. 1 Process landscape for typical USP laser processes arranged by pulse energy and repetition frequency. Diagonal lines of equal average power are marked. Especially the drilling of metallic foils and thin film structuring uses comparable low power $<10 \mathrm{~W}$. Only some applications, e.g. glass cutting and interference patterning utilizes higher power and pulse energy. 


\section{Limitations for Power Scaling}

With pulse durations of $<10 \mathrm{ps}$ and a fluence of $<10$ $\mathrm{J} / \mathrm{cm}^{2}$, copper surfaces and other metal surfaces can be removed in a nearly melt-free way. A surface roughness of Ra $>0.4 \mu \mathrm{m}$ can be achieved in a planar erosion. Thus, through a layer by layer material removal, also gradual three-dimensional shapes can be produced, starting from a digital data set which is transferred to the surface. However, microstructured copper surfaces do not deliver useful properties for all industrial applications. Steel surfaces are often preferred, because they have several advantages compared with other metals, such as hardness, wear resistance, heat resistance and toughness. Moreover, these properties are also individually adjustable. Therefore, it is not surprising that steel is preferably used for tools in stamping and printing applications. If, for these applications, micro-structured surfaces are required, they are currently produced by lithographic technologies, micro drilled or by direct laser ablation in some 100 ns-pulse regime. Micro-structuring of stainless steel X1.4310 and tool steel by means of ultra-short pulsed lasers produces various side effects such as cone-like protrusions (CLPs) or hole formations (Fig. 2), which has been shown by different research groups [5-7].

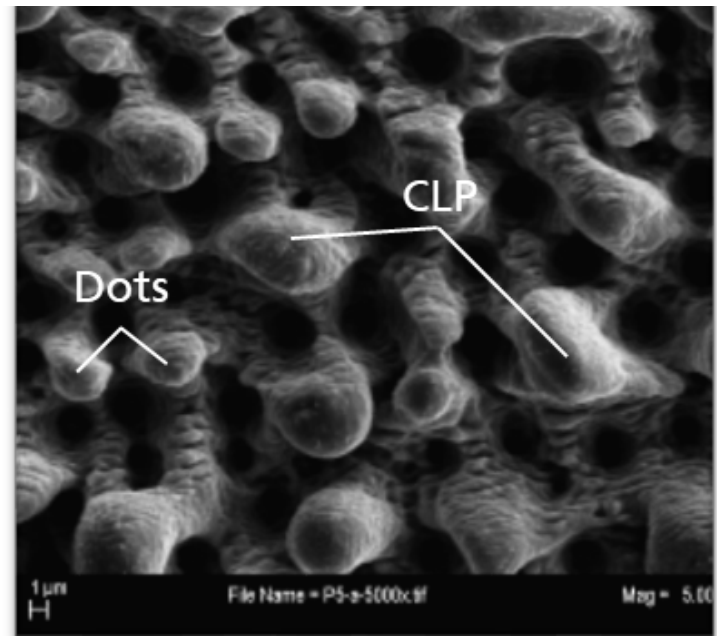

Fig. 2 Laser generated micro dots and cone like protrusions at high laser fluences in steel

These effects can be reduced by a suitable choice of parameters (usually in the range of a fluence in the ablation threshold), but cannot be completely prevented. In the case of stainless steel X 1.4310 and steel St-52, surface defects are emerging on the ablated area like clusters. The formation of the CLP starts at some nucleation of the surface. During a further laser ablation the clusters of CLP are being formed and are growing until the complete area of the ablation zone is covered. The pulse to pulse overlap, adjusted with the surface speed at constant pulse repetition frequency of $2 \mathrm{MHz}$, has an influence on the growth of the CLP clusters. The CLP area in the ablation zone is expanding with decreasing pulse to pulse distance. As a second effect, high average power at low scanning speeds lead to thermal accumulation and subsequent heating by every scan. With a scan to scan repetition rate of several $10 \mathrm{kHz}$ even at metals with good heat conductivity is continuously heated up to the melting point of the material. Thus, the result is material bending, surface melting and reduced ablation quality.

In order to achieve very high ablation qualities the accumulation of surface defects due to laser processing e.g. surface roughness, debris and fluid-dynamical movements of melt must be avoided right from the beginning of the process. This can be achieved by processing layer to layer with the same high quality of the almost cold ablation by ultrashort laser pulses. However, this concept requires an efficient engraving algorithm with well-balanced process parameters to achieve highest precision and maximized ablation rate, which has been evaluated in previous experiments. As a result, it can be stated that in order to preserve the optimized processing conditions at higher power levels, different parameters must be considered to avoid negative effects like plasma absorption, heat accumulation and subsequent melting as well as material redeposition. For instance it has been shown, that the optimum ablation efficiency can be achieved at a certain material specific fluence as shown in Fig. 3 and which has its reason in a specific absorption and energy transfer mechanism as described in [8]. Here the main influencing factor is the optical or accelerated electron penetration depth, the transfer of the energy to the lattice and the energy amount, which is necessary to vaporize the residual material volume. Some basic correlations between pulse energy, fluence, optical penetration depth and ablation efficiency are well described by Neuenschwander et.al in [9].

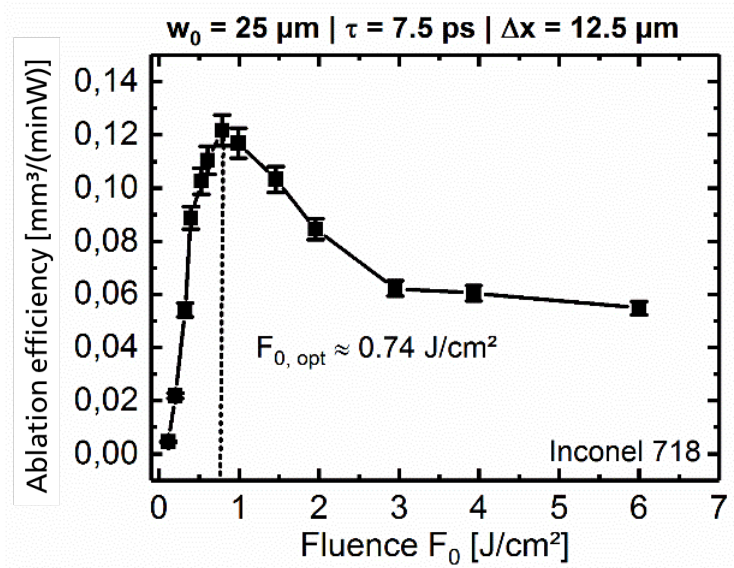

Fig. 3 Characteristic ablation efficiency for Inconel 718 using single pulse picosecond laser radiation

However, this characteristic optimum ablation efficiency depends on certain factors, like pulse duration, the use of pulse bursts, plasma and vapor shielding effects and the absorption and energy transport mechanism of the material.

Moreover, even if the fluence is set to the optimum value, heat accumulation must be controlled to avoid melting of the surface. One possibility to achieve high ablation rates by using high pulse repetition frequencies is to use a polygon mirror scanning system. But even at pulse repetition frequencies of up to $20 \mathrm{MHz}$ and adjusted pulse spacing cone like protrusions with surface roughness of around $1 \mu \mathrm{m}$ are produced [9]. Therefore it is necessary to take also the overall heating process into account, which results from various process parameters. By carefully adjusting pulse repetition frequency, pulse duration, pulse overlap, scan repetition and also numbers of pulses within a pulse burst overheating and melt production can be suppressed [10]. 
The influence of these different effects are currently under investigation by several research groups and are not yet fully understood for the different materials. In an investigation of the influence of the pulse duration on the ablation rate, we have observed a significant increase in ablation efficiency for shorter pulse durations. In laser ablation of steel material, here Inconel 718, the ablation efficiency can be doubled by using a 1 ps pulse duration instead of a longer pulse duration of 8 ps (Fig. 4). The reason for this increase need to be investigated and could be a higher penetration depth of the laser radiation due to lower electron interaction compared to longer pulse durations. Detailed pump and probe measurements at thin metallic films are actually under investigation and will be reported in a following publication.

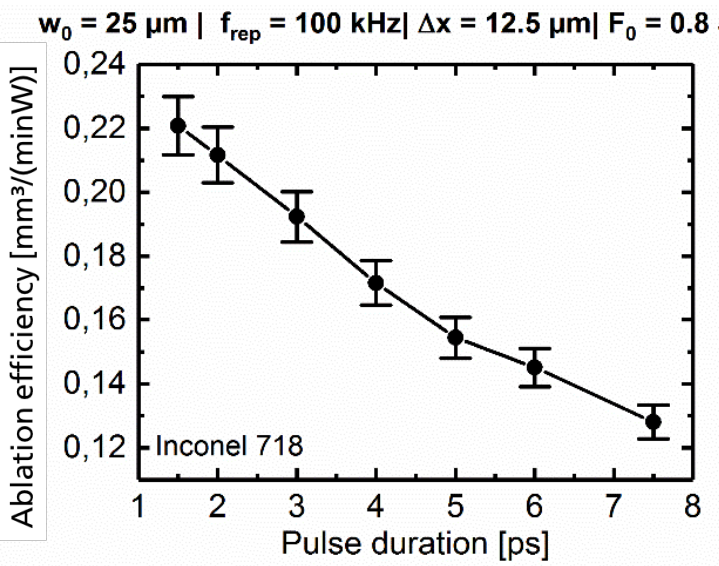

Fig. 4 Characteristic ablation efficiency for Inconel 718 using different pulse durations from $1 \mathrm{ps}$ to $7.5 \mathrm{ps}$

\section{Systems for Power Scaling}

As described above, to avoid negative surface effects like cone like protusions, surface ripples, plasma interaction and thermal accumulation a dedicated system technology together with an adapted processing strategy is necessary. From the current point of view two different approaches can be seen

- Ultrafast scanning with pulse energies with efficient optimized beam fluences and high pulse repetition frequencies

- Multiple beam processing with efficient optimized fluences in each single beam and pulse repetition frequencies in the $100 \mathrm{kHz}$ to $\mathrm{MHz}$-range

Both approaches lead to high average ablation rates and can be used to optimize the usage of high power ultra short pulsed lasers.

\subsection{Ultrafast Scanning Ablation}

The available pulse repetition frequencies of ps-lasers in the $\mathrm{MHz}$ range and the relatively slow scanning speed of common galvanometric scanning devices in the range of 5$10 \mathrm{~m} / \mathrm{s}$ imply a large pulse overlap. This leads to a high local thermal accumulation and a pulse-plasma interaction on the metal surface leading to a decreased machining quality that can be classified between ps- and ns-pulse regimes. An evaluation of the resulting ablation efficiency at different pulseto-pulse overlaps shows, that for a given parameter set of 3.3 $\mathrm{MHz}$ pulse repetition frequency, a spot size of $50 \mu \mathrm{m}$ and a pulse set off of $30 \mu \mathrm{m}$ with a $50 \%$ pulse overlap, the interactions from the following pulses can be neglected. At large overlaps the efficiency drops due to pulse plume interaction and rises again at very large overlaps due to heat accumulation and therefor reduced necessary ablation energy (Fig. 5).

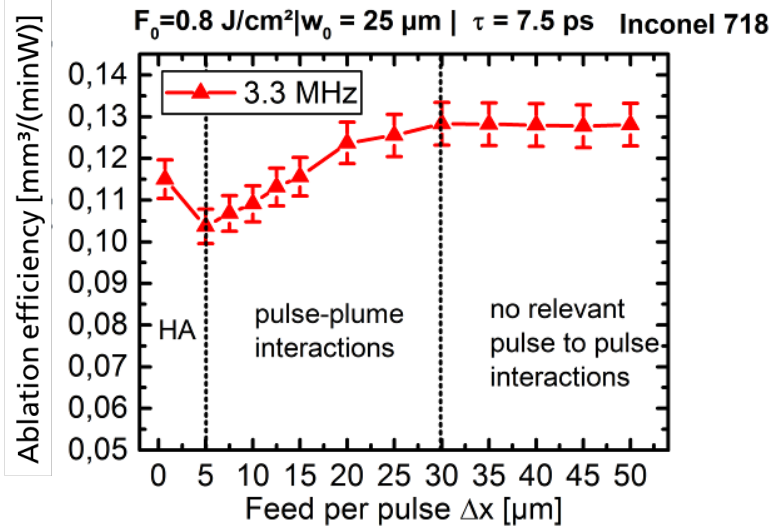

Fig. 5 Characteristic ablation efficiency for Inconel 718 at different pulse overlaps and high pulse repetition frequency of 3.3 $\mathrm{MHz}$

In order to enable the use of high average laser power by high pulse repetition frequencies and resulting high productivity with less thermal effects, an ultrafast scanning technique is required. One solution for this is the use of a polygon mirror device. For high scanning speeds in the range> $100 \mathrm{~m} / \mathrm{s}$ and a fast material removal with high repetition ultrashort pulse lasers a processing system with a fast polygon scanner and a fast beam modulator was realized. For polygon scanners a polygon mirror rotating at high, constant speed of multi $10 \mathrm{~Hz} / \mathrm{s}$ was developed thereby increasing the maximum processing speed significantly. The optics is based on a high speed drive in combination with a multi facet mirror system. A conventional f-Theta-Optics can be used for a processing field of up to $100 \mathrm{~mm}$ with $160 \mathrm{~mm}$ optics and a scan speed of up to $320 \mathrm{~m} / \mathrm{s}$. The modulation of the laser beam at several $\mathrm{MHz}$ in synchronisation with the laser cycle and the correspondingly adjusted positioning of the laser beam on the workpiece was realized by using a fast FPGA device, which controls the laser unit according to the position of the multifacet polygon mirror. By using this technology every single laser pulse can set with a low pulse overlap for optimal results when utilizing the full laser power.

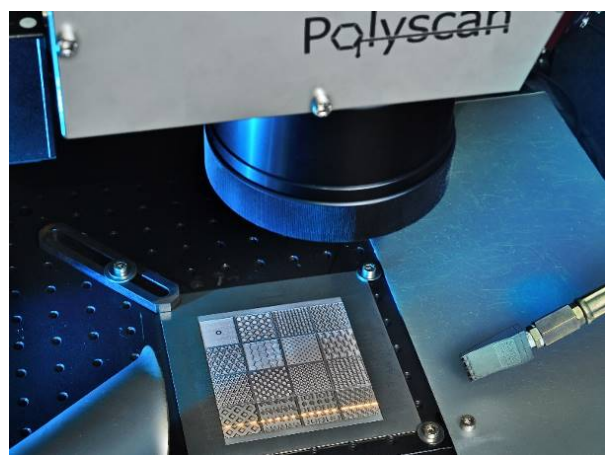

Fig. 6 Ultrafast polygon scanning device for high power ultrafast laser processing at processing speed larger $300 \mathrm{~m} / \mathrm{s}$ and spot size $<30 \mu \mathrm{m}$

For processing a large area, the laser beam is deflected along a line and by displacement of this line a two-dimensional processing is carried out with a high accuracy moving system. In this way the entire workpiece can be processed in 
a bitmap mode. The system has been integrated into a high precision laser cutting machine for semiconductors. In Fig. 6 the system is shown for surface machining of large tools for the generation of functional surfaces, like super hydrophobicity, special optical effects and improved wear properties, see also Fig. 7.

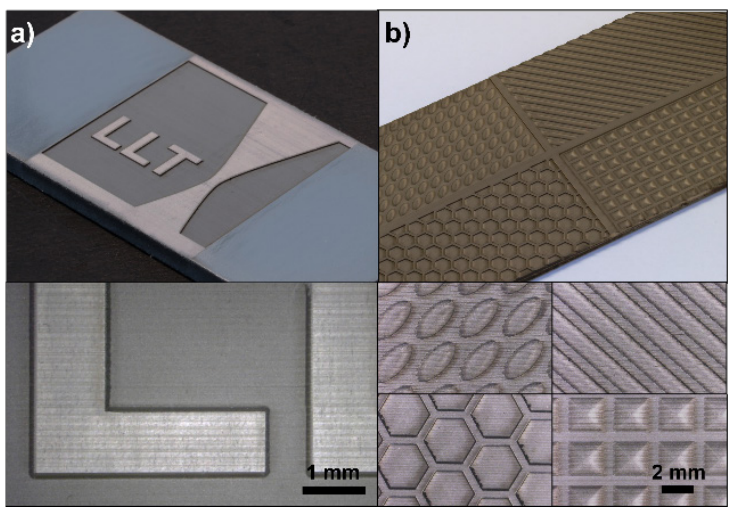

Fig. 7 Results of USP processing of Inconel 718 with high repetition frequency and average power. By using a polygon-scanner for fast beam deflection, relevant affection of the surface quality by heat accumulation can be avoided. The average surface rough-ness is $\mathrm{Ra}<2 \mu \mathrm{m}$.

a) Ablation rate $18.5 \mathrm{~mm}^{3} / \mathrm{min}$ using $\mathrm{P}=140 \mathrm{~W}$, frep $=14400$ $\mathrm{kHz}, \Delta \mathrm{x}=21 \mu \mathrm{m}, 2 \mathrm{w} 0=50 \mu \mathrm{m}$

b) Ablation rate $32 \mathrm{~mm}^{3} / \mathrm{min}$ using $\mathrm{P}=210 \mathrm{~W}$, frep $=18900$ $\mathrm{kHz}, \Delta \mathrm{x}=20 \mu \mathrm{m}, 2 \mathrm{w} 0=50 \mu \mathrm{m}$

\subsection{Multi Beam Ablation}

The second approach to increase productivity and overall process efficiency is the use of multibeams with moderate laser fluence for the optimum characteristic ablation efficiency. With system setup having beam splitting devices to enable parallel processing with multiple beams at low energy levels of each single beam, the productivity simply scales with the number of single laser beams. By this approach the utilization of ultrashort pulsed laser sources with high average power is possible up to the kw-range using hundreds of individual laser beams simultaneously. The input beam is divided in a bundle of individual beams by either a static diffractive, optical element (DOE) or a dynamic, spatial light modulator (SLM). Other technologies like "Grating light valve" $[7,8]$ and piezo-electric mirrors [9-11] are limited in their applications.

However shaping and steering of multiple beams requires particular optical systems which are not state of the art today. Limitations for large spot arrays are evaluated and considered for the design concept of appropriate optical systems.

\subsubsection{Static Diffractive Optical Elements}

For the purpose of micro structuring with high demands on the spatial accuracy, an optical system based on a diffractive optical beam splitter is designed and setup. By splitting the laser beam into several beams, significantly greater laser power can be utilized just by multiplying the number of beams. This beam splitting is made possible by a diffractive optical element (DOE). The DOE consists of an array of microstructures, which are capable of producing virtually any type of intensity distribution behind the element by means of diffraction - depending on the design (Fig. 8).

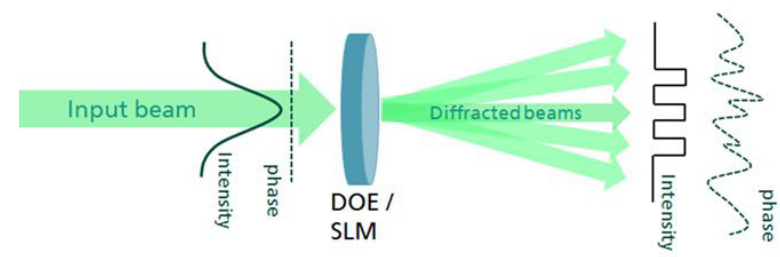

Fig. 8 Principle setup of beam splitting by a diffractive optical element DOE

The DOE is integrated into an optical setup between the beam source and a galvanometer scanner in such a way that the split laser beams are imaged in the galvanometer scanner by a multiple relay lens system. The setup, as shown in Fig. 9, consists of a beam expanding unit, which enlarges the incoming laser beam to the size of the DOE. The diffracted laser beam is then imaged by a first relay lens system into a zoom optics, which allows the variation of the distances of the single laser beams in $\mathrm{x}$ - and $\mathrm{y}$-direction. With a second relay lens system the entire array is then imaged into the input aperture of a conventional scanner system to allow large field scanning of the array across the workpiece. The relay lens systems are designed in the way, that they are collecting all diffracted laser beams with a minimum of distortion of the beam arrays by using a combination of spheric and aspheric Quartz lenses.

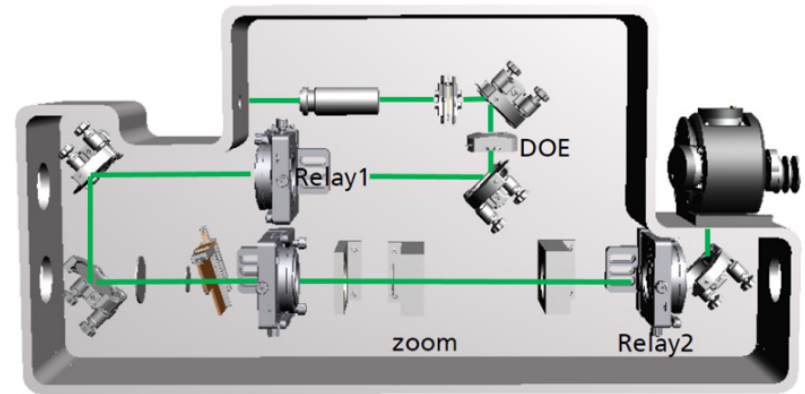

Fig. 9 Optical system for beam splitting by DOE and scanning the beam bundle with a galvo scanner

Furthermore, this relay lens system offers a practicable solution to remove higher diffraction orders of the DOE. From the basic principle, this DOE-setup produces a periodic pattern of laser beams, where numbers of beams and distances of beam remain static and modulation and switching of laser lead to modulation of all generated single laser beams. However this bundle of beams can be handled as a single laser beam without losing the beam quality of each single beam. Using this fact, the beam bundle can be guided through a conventional galvo system and scanned across the surface of the workpiece.

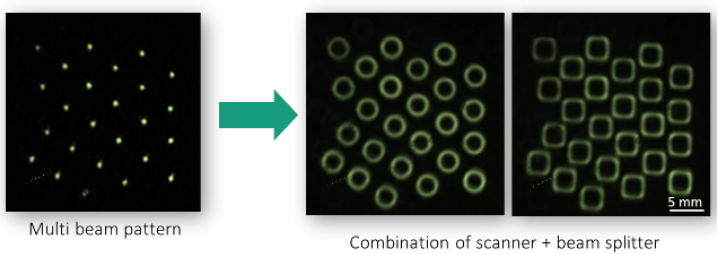

Fig 10 Arbitrary scanned pattern using a combination of static DOE and scanning the beam bundle with a galvo scanner 
Focusing the beams using an $\mathrm{f}$-theta lens produces a periodic array of processing points with identic laser parameters. With this setup it possible to ablate periodic patterns of any complexity within a single irradiation area even on a large scale (see Fig. 10). Due to the scanner a highly dynamic, simultaneous deflection of all partial laser beams can be achieved and controlled arbitrary. In the presented setup all generated single laser beams are modulated and switched parallel. Newer developments however allow the switching of each single laser beam from the DOE to allow even higher flexibility on surface patterning, shown in the following paragraphs.

However, with the implementation of a highly parallel beam system, several optical issues have to be taken in account. First, the focusing system is not ideally adapted to the focusing of a beam array. Setting up an x-y-beam with a DOE results in spot distances, which follow the following equation:

$$
\mathrm{D}=\mathrm{WD} * \tan (\theta)
$$

\section{WD: Working distance $=$ focal length}

\section{$\theta$ : Separation angle of diffraction order}

Therefor the real positions of the single spots differ from a desired rectangular array (Fig. 11). To compensate this nonlinear effect, a special design of the DOE has to be chosen, in which the diffraction angle is modified by higher orders. Using this approach spot positioning errors of less than 1 micrometer at a $100 \mathrm{~mm}$ focal length have been achieved. For the alignment and the experimental evaluation of the complex optical system appropriate measurement devices are necessary. The simultaneous determination of several spot positions is realized by a camera system and adapted evaluation software.
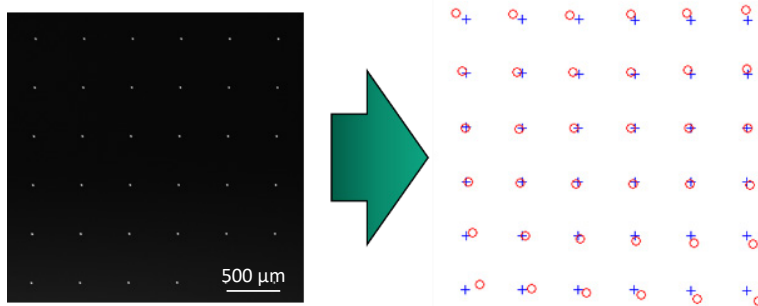

Fig 11 Spot array and deviation of spots after focusing with a $100 \mathrm{~mm}$ lens (circles indicate spot positions), deviation not to scale $<1 \mu \mathrm{m}$

Furthermore scanning the entire beam bundle also result in a deviation of ablation pattern. In a normal scanner application the single spot position is calibrated to the used lens and scanner by slightly modification of the scanning angle. In this way an accurate positioning of the laser beam can be achieved across the entire scanning field. In multi beam scanning the position of the entire array also can be corrected with an appropriate calibration file as indicated in Fig. 12 (left). However, the shape of the array cannot be compensated by this technology. Here a special lens design has to be chosen, which follows a f-sin(Theta) approach instead of a
f-Theta-principle (Fig. 12 right) [12]. An exact compensation can be achieved using a special compensation scanner unit, which is synchronized to the galvo scanner unit.
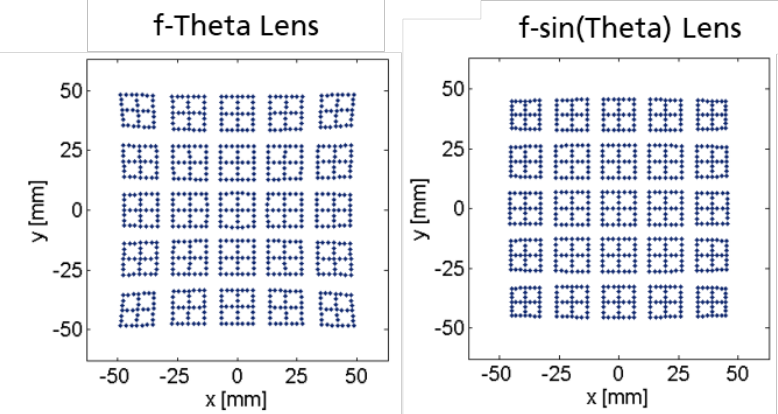

Fig. 12 Simulated beam pattern array after scanning with two lens principles, left: f-theta lens, right: f-sin(Theta) lens

The splitting of a laser beam into 196 beamlets has already been successfully demonstrated in an optical setup and has been integrated in a laser processing machine. This beam parallelization enables the workpiece to be processed at 196 periodically arranged points simultaneously, resulting in an almost 200 -fold increase in processing speed compared to a single laser beam.

In further developments the static DOE system is now modified with single beam attenuators and fast beam switching for each single beam. Fig. 13 shows a first setup for 4 single beams, in which all beams coming from a DOE are guided through an acoustooptic beam switch and can be switched on and off according to the desired ablation pattern. With this device and a high power ps-laser micro drilling and micro ablation can be used to generate arbitrary filter applications and thin film ablation as shown in section 5 .

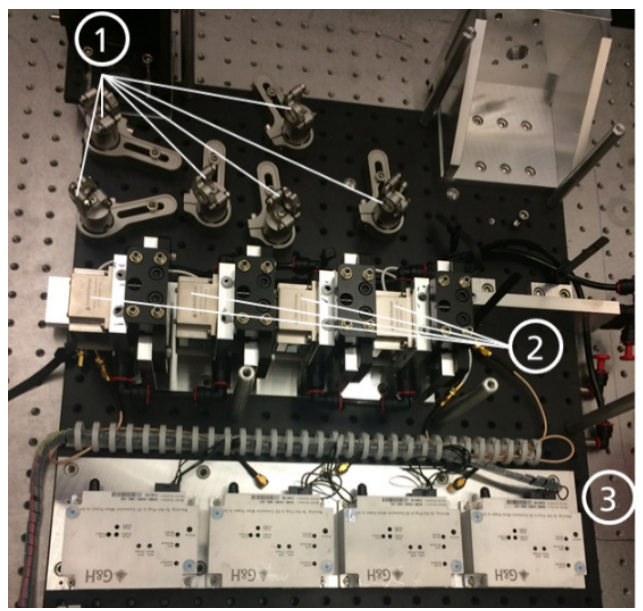

Fig. 13 Modular beam splitting and beam switching unit for multi parallel ultrafast laser processing.

1: Beam bending mirrors, 2: Acoustooptic modulators, 3: Controllers

\subsubsection{Programmable Diffractive Optical Elements}

A more flexible approach with respect to the spot configuration and pattern shape is the use of a spatial light modulator (SLM) which is placed in front of a galvanometric scanning system and which forms together with the optical components a newly designed Programmable Diffractive Optics (PDO). The PDO works similar to the static DOEdevices but allows flexible modulating amplitude, phase or 
than the ablation process. Taking the hole shape and the deposited energy into account, the temperature distribution is calculated over time.

Subsequently the model can be expanded towards a multi-beam model. The energy distribution among the different beams of the pattern is then taken into account.

If there is a heat flux from multiple laser spots, then the beams in the center of a beam matrix may be subjected to increased temperatures on the material compared to beams on the outer circle, leading to different processing results and to increased distortion. Furthermore, the thermal and mechanical properties of the material change with each piece of material removed. To be able to calculate the temperature and the distortion on a macroscopic scale a two-scale approach [16] is applied. The heat flux from multiple holes processed in parallel is included and effective material properties are calculated for Representative Volume Elements describing the properties of the ablated structures. In order to simulate the processing of larger workpieces a macro model of the work piece is created involving the effects of all beams as one unified heat source. Precalculated effective material properties and effective heat sources are used. Therewith it becomes possible to build a heat map over the total workpiece and to calculate the resulting distortion.

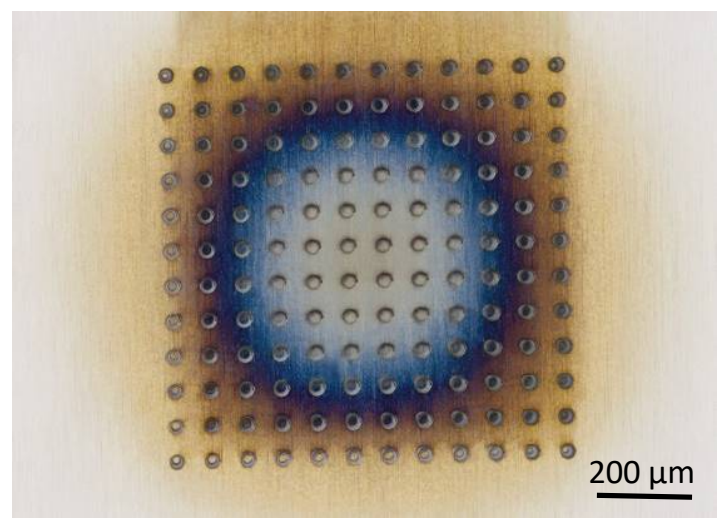

Fig. 18 Thermal flux between different holes of a pattern may lead to increased heat load in the center of the pattern. The annealing color in the center corresponds to a temperature of about $360^{\circ} \mathrm{C}(630 \mathrm{~K})$.

After all, the developed simulation tool helps users to understand, which parameter is the knob to turn for a systematically optimized result. The simulation tool can be used to evaluate the chosen process parameter's influence and helps to identify the reasons for insufficient quality in process results such as shown in Fig. 18. There, the heat flow between the single spots is much too large and there might be substantial tensions as well. Fig. 19 shows the simulation result for such a process and heat as well as tension effects are well reproduced. Fig. 20 shows a comparison between experimental and simulation results for a multi-beam process. Ablation structures calculated with the micro-model are in good agreement with experimental observed structures.

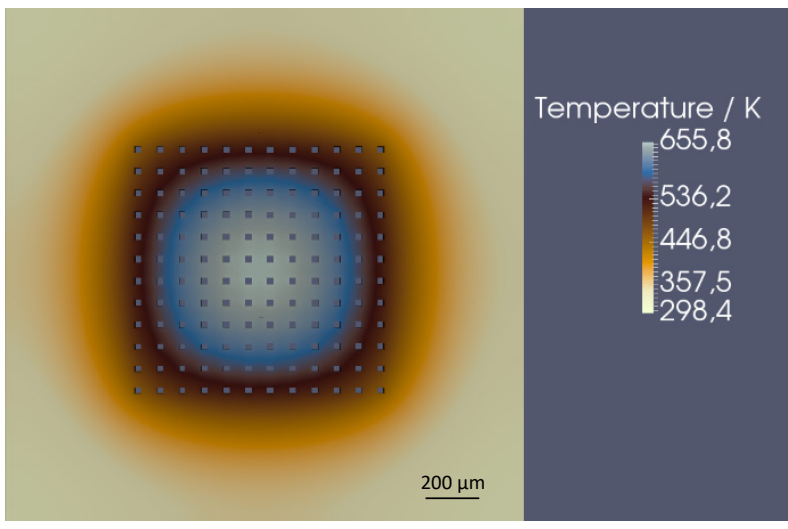

Fig 19 A simulation with one shot on $12 \times 12$ beams with 100 $\mu \mathrm{m}$ distance shows that the thermal load is not homogeneous.

The level of heating agrees well with that of the experiment shown in Fig. 18.

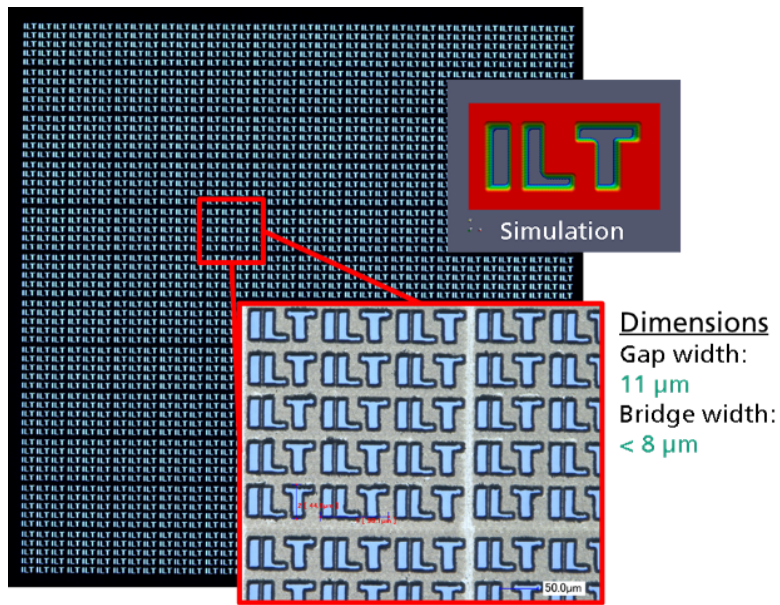

Fig 20 Comparison between experimental and simulation results for a multi beam processing

Within an optimization loop various parameters can be optimized. There are pulse parameters, such as pulse energy, spot diameter or repetition rate. Then there are parameters of the pattern such as the spot distribution and the energy distribution among the spots. Also different optics or filters can be introduced on that point. Finally, there might be additional process parameters such as cooling or process gas that can be added to optimize the simulation result. A simulation tool has been developed that is capable of simulating multi beam as well as high speed scanning scenarios or combinations of both. It may help prospective users of ultrafast lasers for material processing in two ways: In the phase of investment planning, the simulation may help to find out which range of machine parameters would be required for a typical range of applications.

\section{Experimental Results}

For materials processing with USP laser radiation with high pulse energy multi-beam processing is an effective approach to homogeneously distribute the pulse energy across the surface of the workpiece without losing the high resolution of materials processing by means of USP laser radiation with small focal size. Here, every single laser process is tuned to the working point of highest efficiency and multiplied by the number of beams. In this manner, the throughput 
for the generation of accurate periodic structures can be significantly increased. Moreover, with the use of multiple laser spots high average laser power can be applied even for pulse repetition frequencies that are small enough to limit the negative effects of heat accumulation.

However, in addition to the aforementioned effects, the heat-input of the multi-beam pattern during materials processing leads to an additional temperature rise that can affect the processing results.

In Fig. 21 a), the result of percussion drilling of a thin steel foil with a 12 times 12 beam array, generated by a static DOE, is shown. As a comparison in Fig. 21 b) the simulation of the entire process shows the same characteristics. Therefor the multi scale simulation is used to optimize the multi beam drilling process with respect to critical process parameters, like fluence, scanning strategy, repetition frequency and spot size. The interaction between the heat sources within the spot array leads to location dependent, macroscopic thermal input that leads to the generation of annealing colors in the shown case. For smaller structures, such thermal spot-to-spot interaction leads to burrs and recast when processing thermal sensitive materials as shown in Fig. 21 c). A simulation of the shown process is in need of a multi scale approach as presented by Finger et al. [11]. The complete steps from heat input of a single laser pulse to the heat during drilling a hole and up to the thermal interaction between the different spots within one array have to be taken into account as shown in Fig. $21 \mathrm{~d}$ ). For a homogenous processing result, the heat accumulation of a multi laser foci pattern needs to be controlled by processing strategies and the distance of the laser beams. However, one of the most important factor to avoid strong heat accumulation is the choice of efficient processing parameters for each single beam to limit the heat-input right from the start.

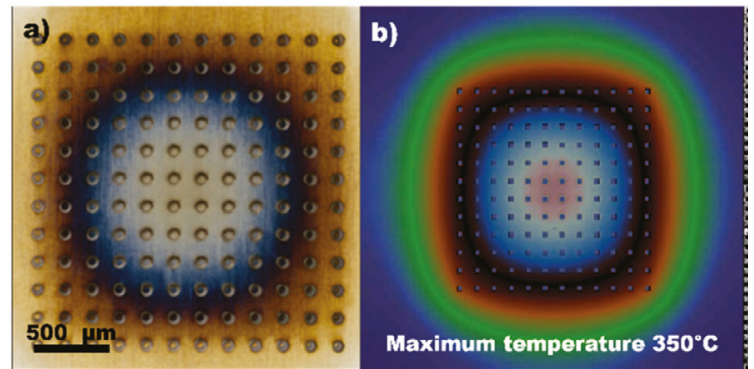

Fig. 21 a and b Comparison between experimental and simulation results for multi beam percussing drilling with $12 \times 12$ beam array

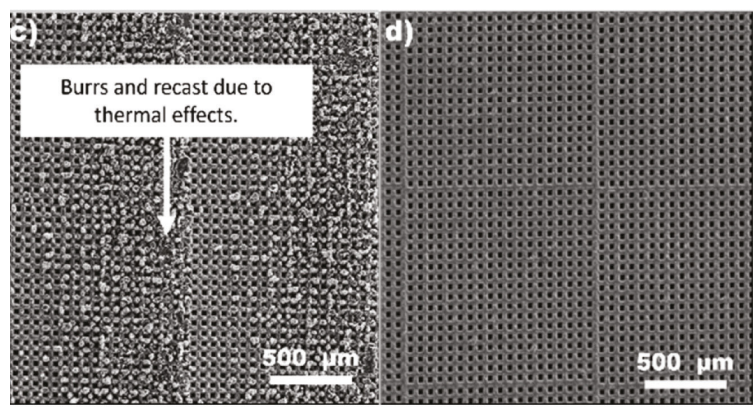

Fig. $21 \mathrm{c}$ and d: Not optimized laser drilling with thermal effects and generation of recast and burrs (c). optimized laser drilling after simulation based matching of process parameters (d)
The described technology and system setups allow scaling of the productivity in ultrashort pulsed laser processing by fast scanning and multiple laser beams. Especially multi beam approaches offer the possibility, to increase productivity, while keeping the quality of ultrafast laser ablation. Currently most multi beam applications are based on static DOEs and are used for drilling micro filters, where periodic patterns are needed. With the combination of DOEs with a fast galvo scanner even arbitrary shaped patterns can be generated as indicated in Fig. 22. High precision scanners allow exact structuring within accuracies of less than 1 micrometer.

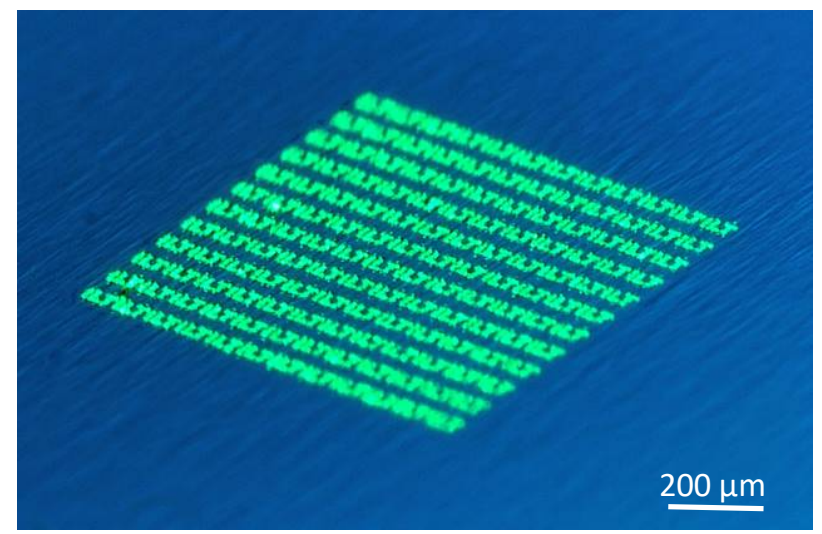

Fig. 22 Multi beam ablation with a combination of DOEs and galvo scanner

With the combination of the shown optical system and high power ultrafast lasers, the generation of highly specific micro filters in metal foils are possible. In Fig. 23 an example of a metal filter with hole dimensions of less than $10 \mathrm{mi}-$ crometer is shown. The magnification in the lower left part of the figure shows the accuracy of the single holes.

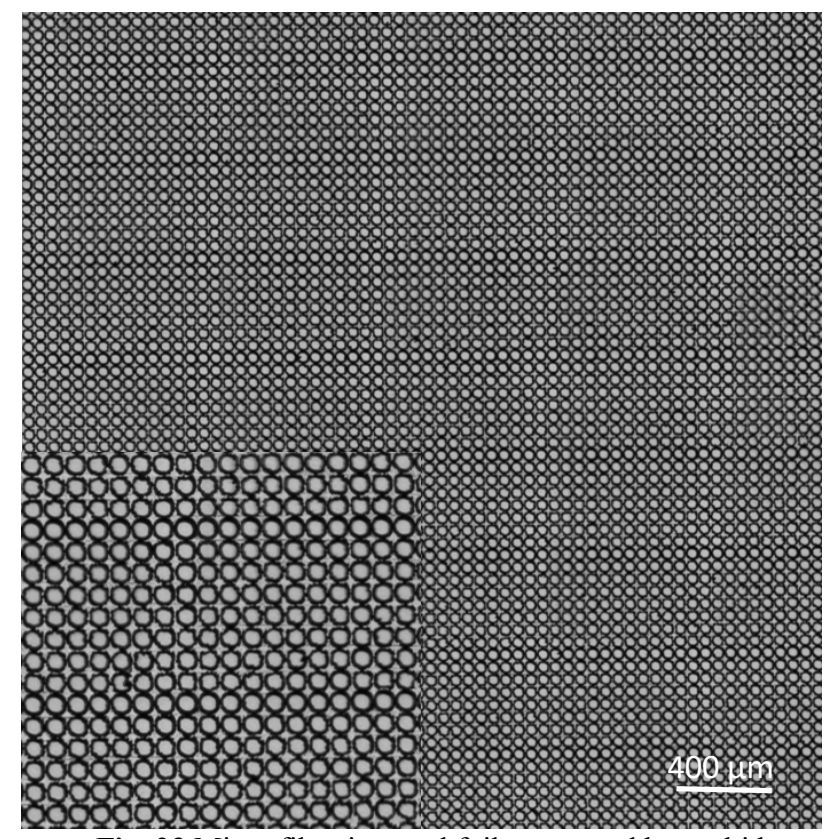

Fig. 23 Micro filter in metal foil, generated by multi beam ablation with a combination of DOEs and galvo scanner, spot distance $50 \mu \mathrm{m}$ 


\section{Conclusion}

Using two different scanning techniques, the potential of high power ultrashort pulsed laser ablation of metal surfaces for respect to ablation quality and efficiency has been investigated with different approaches. Since high fluences produce negative effects such as cone like protusions and thermal accumulations, a large pulse to pulse overlap as well as high fluences have to be avoided. At high pulse frequencies with high pulse overlap, an increase of thermal effects (debris, molten parts, and oxide layers) can be seen. This can be reduced by ultra high speed scanning using polygon scanners. As an alternative the use of diffractive optical elements (DOE) with a significant reduction of fluence in each single laser beam can be used with a very high overall efficiency of using the available laser power of more than $90 \%$. Using DOEs with several hundred laser beams, the productivity drastically can therefore be significantly increased. Moreover with spatial light modulators (SLM) new technologies are available to change interference patterns thus allowing large field processing with ultimate ablation quality.

In future, this technology will permit the output reserves of current high-power ultra-short-pulse laser systems to be fully utilized on the workpiece for ultrashort pulse laser processing. Processing times will drop accordingly, leading to a significant reduction in overall process costs. This will make USP lasers significantly more attractive to users from an economic point of view for manufacturing periodic microstructures. With this approach, it becomes economically feasible to structure even large surfaces. The long-term goal is to use multi-hundred-watt lasers for micro structuring before too long.

\section{Acknowledgment}

Part of this work has been financed by the German Federal Ministry of Education and Research for supporting the development of high precision ps-ablation within the project MULTISURF.

\section{References}

[1] T. Eidam, S. Hanf, E. Seise, T. Andersen, T. Gabler, C. Wirth, T. Schreiber, J. Limpert, A. Tünnermann: Optics Letters, 35, (2010) 94.

[2] P. Russbueldt, T. Mans, G. Rotarius, J. Weitenberg, H.D. Hoffmann, R. Poprawe: Optics Express, 17, (2019) 12230.

[3] P. Russbüldt, T. Mans, J. Weitenberg, H. D. Hoffmann, and R. Poprawe: Optics Letters, 35, (2010) 4169.

[4] J. Negel, A. Voss, M. Ahmed, D. Bauer, D. Sutter, A. Killi, T. Graf: Optics Letters, 38, (2013) 5442.

[5] M.Tsukamoto , T. Kayahara , H. Nakano, M. Hashida, M. Katto, M. Fujita, M. Tanaka, and N. Abe:, Journal of Physics: Conference Series, 59, (2007) 666.

[6] V. Zorba, E. Stratakis, M. Barberoglou, E. Spanakis, P. Tzanetakis, C. Fotakis: Appl Phys A, 93, (2008) 819.

[7] J. Schille, R. Ebert, U. Loeschner, P. Regenfuss, T. Suess, H. Exner: Proceedings of LPM2008, Quebec 2008

[8] B. Jaeggi, S. Remund, R. Streubel, B. Goekce, S. Barcikowski, B. Neuenschwander: Journal of Laser Micro Nanoengineering, 12 (2017) 267.
[9] B. Neuenschwander, B. Jaeggi, M. Schmid, V. Rouffiange, P. Martin: Proc. of Laser Applications in Microelectronic and Optoelectronic Manufacturing (LAMOM) XVII, Proc. SPIE 8243 (2012)

[10] J. Schille, L. Schneider, A. Streek, S. Kloetzer, and U. Loeschner: Optical Engineering 55, (2016) 096109

[11] J. Finger, B. Bornschlegel, M. Reininghaus, A. Dohrn, M. Nießen, A. Gillner, R. Poprawe:, Advanced Optical Technologies, 7, (2018) 145

[12]Z. Tong, W. Shen, S. Song, W. Cheng, Z. Cai, Y. Ma, L. Wei, W. Ma, L. Xiao, S. Jia: Optics Express 25, (2017) 3795

[13]D. M. Bloom: Proc. of Electronic Imaging '97, Proc. SPIE 3013 (1997) 165

[14] D. Spiga, L. Raimondi, C. Svetina, and M. Zangrando: Nuclear Instruments and Methods in Physics Research Section A: Accelerators, Spectrometers, Detectors and Associated Equipment, 710, (2013) 125

[15] C. Svetina, G. Sostero, R. Sergo, R. Borghes, C. Callegari, F. Damico, F. Bencivenga, C. Masciovecchio, A. Di Cicco, and D. Cocco: Nuclear Instruments and Methods in Physics Research Section A: Accelerators, Spectrometers, Detectors and Associated Equipment 635, (2011), 12

[16] K. L. Wlodarczyk, E. Bryce, N. Schwartz, M. Strachan, D. Hutson, R. R. Maier, D. Atkinson, S. Beard, T. Baillie, P. Parr-Burman: Review of Scientific Instruments 85, (2014) 024502

[17]L. Büsing, S. Eifel, P. Loosen: Proc. of Optical Modelling and Design 2014, Proc. SPIE 9131 (2014)

[18]E. G. Gamaly, A. V. Rode, B. Luther-Davies: Physics of Plasmas 9 (2002) 949.

[19] S. Osher and R. Fedkiw: Applied Mathematical Sciences, 153 (2003)

[20] J. Fish: "Practical Multiscaling", Wiley 2013

(Received: August 7, 2018, Accepted: April 28, 2019) 